\title{
De la marginalité territoriale à la recomposition territoriale « marginale »
}

From territorial marginality to marginal territorial recomposition

Von der territorialen Marginalität zur marginalen territorialen Neuordnung

\section{Sophie de Ruffray}

\section{(2) OpenEdition \\ Journals}

Édition électronique

URL : http://journals.openedition.org/rge/4061

DOI : $10.4000 /$ rge.4061

ISSN : $2108-6478$

Éditeur

Association des géographes de l'Est

Édition imprimée

Date de publication : 1 septembre 2000

ISSN : 0035-3213

\section{Référence électronique}

Sophie de Ruffray, « De la marginalité territoriale à la recomposition territoriale « marginale » », Revue Géographique de l'Est [En ligne], vol. 40 / 4 | 2000, mis en ligne le 27 juillet 2013, consulté le 08 septembre 2020. URL : http://journals.openedition.org/rge/4061 ; DOI : https://doi.org/10.4000/rge. 4061

Ce document a été généré automatiquement le 8 septembre 2020

Tous droits réservés 


\title{
De la marginalité territoriale à la recomposition territoriale « marginale »
}

\author{
From territorial marginality to marginal territorial recomposition \\ Von der territorialen Marginalität zur marginalen territorialen Neuordnung
}

Sophie de Ruffray

\section{Introduction}

1 Dans le contexte actuel des recompositions territoriales (Loi d'orientation pour l'aménagement et le développement durable du territoire de juin 1999, Loi relative au renforcement et à la simplification de la coopération intercommunale de juillet 1999), les territoires en marges des systèmes régionaux, déjà sensibilisés aux dynamiques interrégionales, méritent l'attention particulière du chercheur et du décideur, tant ils constituent des situations complexes et des enjeux pour l'aménagement.

Cet article a pour objectif de contribuer à la réflexion sur cette problématique de recomposition des territoires de marges en présentant des modèles de recomposition "marginale", fondé sur des interfaces particulières: l'organisation des petites villes en réseau. Sur le plan conceptuel, cette affirmation prend appui sur la notion de système territorial global dans lequel les concepts de territoire et de réseau sont étroitement associés. Sur le plan territorial, elle se réfère à des travaux menés dans l'espace interrégional, en situation d'interface entre les confins orientaux de la Moselle et nordoccidentaux du Bas-Rhin. Avec près de 278000 habitants, il s'agit d'un espace relativement homogène en termes de contraintes physiques, de ruralité industrieuse ancienne, d'éloignement des métropoles régionales Metz et Strasbourg. Cet espace a, en plus, subi les aléas de "frontières" multiples (linguistiques, religieuses, politiques, administratives)... 
3 Notre démarche s'articule autour de la mise en convergence des propriétés d'un système marginal et de la problématique d'une recomposition territoriale. Nous explicitons ensuite la recomposition à l'échelle des acteurs; en dernier lieu, nous proposons un modèle de recomposition marginale, fondé sur une configuration de villes en réseau.

\section{Système territorial marginal et recomposition territoriale : spécificités de la mise en convergence}

Les concepts de marges et de marginalité doivent, avant toute démarche, être explicités en vue de proposer notre définition d'une recomposition marginale du territoire. Les spécificités de la mise en convergence entre le système territorial et la recomposition territoriale reposent sur la complexité de la définition et sur les propriétés intrinsèques d'un système marginal.

\section{A. Une définition complexe d'un système de marges}

5 Couramment utilisé dans des champs scientifiques liés aux sciences sociales, le terme de marges évoque tout à la fois des aspects positifs et négatifs. Ainsi, d'un point de vue historique, la marge est un espace blanc autour d'une page de texte écrit ou imprimé, transformé en bord ou bordure. Le sens littéral évoque pour le géographe un espace "interstitiel», aux limites d'influence... Les sens dérivés désignent à la fois un intervalle d'espace ou de temps et une possibilité d'action. En tout cas, un espace marginal est situé, par définition, loin du centre, n'évoquant pas seulement une distance physique mais aussi une distance psychologique, puisqu'il signifie aussi l'accessoire, le secondaire.

6 Implicitement, bon nombre d'auteurs se réfèrent aux concepts de centre et de périphérie pour proposer des définitions et des localisations de systèmes régionaux ou locaux. Ainsi, pour A. Reynaud (1992), tout territoire, quelle que soit l'échelle spatiale considérée, se subdivise en sous-ensembles entre lesquels existent des inégalités de développement. Ainsi, c'est le concept de flux qui sous-tend la notion de marges : si un sous-ensemble territorial n'entretient pas de relations avec l'extérieur et vit pratiquement de ses seules ressources, il constitue un isolat. En fait, le plus souvent, des relations existent entre sous-ensembles spatiaux - flux d'hommes, flux de marchandises, de capitaux et d'informations, qu'il est indispensable de prendre en compte. Les flux présentent souvent une dissymétrie, que l'on résume souvent par l'opposition centre-périphérie.

7 La plupart des géographes considèrent que le centre est " là où les choses se passent ", c'est-à-dire une certaine masse de population, une capacité notable de production, un niveau de vie élevé, associé aussi à l'idée de pouvoir et d'une capacité d'innovation. A l'opposé, la périphérie se définit négativement par rapport au centre, l'un des principaux handicaps résidant dans l'absence d'autonomie en matière décisionnelle. Un territoire de marges se définit alors comme une périphérie particulière, à une distance réelle ou perçue de centres régionaux.

$8 \mathrm{Si}$, dans la plupart des cas, le concept «centre-périphérie » explique les phénomènes observés, les espaces de marges nécessitent d'avoir recours à d'autres concepts. Comme 
le montre le tableau 1, un espace de marges possède à la fois des propriétés du centre (capacité d'organisation et d'innovation) et de la périphérie (degré de dépendance notamment en ce qui concerne les activités économiques, qui se traduit par des flux domicile-travail...). Ensemble d'éléments en interaction dynamique, un espace de marges se définit en toute généralité comme un système territorial, où les quatre concepts fondamentaux posés par J. de Rosnay (1975) se retrouvent : l'interaction entre le système et son environnement, la globalité, l'organisation et enfin la complexité. Ce système revêt, dans le cas qui nous intéresse ici, des propriétés particulières. Ainsi, C. Rolland-May (2001) définit "un système territorial de marges" comme une frange territoriale possédant des propriétés systémiques, mais dont la fermeture relative réserve l'accès à quelques portes, maintenant le système à un état quasi insulaire, dont certaines caractéristiques fortes traduisent l'enclosure. Outre le nivellement des différents niveaux de complexité interne, le système de marges, tout en se fermant sur son environnement, reste demandeur et importateur d'énergie exogène nécessaire à sa pérennité, pour qu'il se transforme tout en restant globalement invariant... Il en résulte qu'un système territorial de marges est une interface, dispositif destiné à assurer la connexion entre deux systèmes voisins, zone de contact plus ou moins large, plus ou moins "poreuse", entre deux systèmes distincts. Une interface est aussi un lieu d'interaction entre deux systèmes, deux organisations; des phénomènes originaux s'y produisent, qui concernent à la fois des échanges ou des modifications entre l'un et l'autre système.

Tableau 1 : Une définition ou des définitions d'un espace de marges

\begin{tabular}{|c|c|c|c|}
\hline Mots-clés & Définitions & Propriétés & $\begin{array}{c}\text { Conséquences } \\
\text { pour un espace de marges }\end{array}$ \\
\hline MARGINALITE & $\begin{array}{l}\text { "Caractère de ce qui ne fait pas } \\
\text { pleinement partie du système " } \\
\text { "Le marginal est parfois toléré, } \\
\text { parfois récupéré, parfois combattu } \\
\text { par le système " } \\
\text { "La marginalité peut être féconde } \\
\text { par sa capacité à produire } \\
\text { des visions originales » }\end{array}$ & $\begin{array}{l}\text { Mauvaise intégration } \\
\text { - à l'échelle régionale } \\
\text { - à l'échelle locale }\end{array}$ & \multirow{3}{*}{$\begin{array}{l}\text { L'espace de marges possède } \\
\text { des propriétés systémiques } \\
\text { (ouverture plus ou moins grande } \\
\text { du système avec son environnement, } \\
\text { capacités de fonctionnement } \\
\text { (résistance, ajustement, } \\
\text { auto-apprentissage) particulières: } \\
\text { - limites plus ou moins nettes } \\
\text { du système avec son environnement } \\
\text { (identité...) } \\
\text { - mauvaise intégration du système } \\
\text { à son environnement (notamment } \\
\text { à travers le réseau urbain) } \\
\text { - degré d'autonomie } \\
\text { et d'indépendance } \\
\text { - capacité d'organisation } \\
\text { et d'innovation }\end{array}$} \\
\hline MARGES & $\begin{array}{l}\text { "Bordure, limite dotée de quelque } \\
\text { épaisseur et considérée comme } \\
\text { en position de subordination " } \\
\text { - blanc, vide } \\
\text { - a de la liberté dans l'espace } \\
\text { et dans le temps } \\
\text { "Un espace, qui au cours du temps, } \\
\text { intègre des zones successives de } \\
\text { l'environnement ou au contraire, } \\
\text { abandonne ses éléments propres " }\end{array}$ & $\begin{array}{l}\text { Degré d'autonomie voire } \\
\text { d'indépendance } \\
\text { Limites nettes, variables } \\
\text { dans l'espace et dans le temps }\end{array}$ & \\
\hline MARCHES & $\begin{array}{l}\text { «no man's land marquant } \\
\text { une séparation nette entre deux } \\
\text { territoires définis " } \\
\text { "Zone périphérique, délaissée } \\
\text { d'un système, soumise à lautorité } \\
\text { directe comme lieu d'affirmation } \\
\text { du pouvoir mais qui, compte tenu } \\
\text { de ses caractéristiques indépendantes, } \\
\text { a de la liberté dans l'espace } \\
\text { et dans le temps " } \\
\text { "Espace tampon, espaces tenus mais } \\
\text { non encore incorporés, espaces de } \\
\text { protection et d'expérimentation, avec } \\
\text { des populations tantôt insoumises, } \\
\text { tantôt ignorées ou utilisées \# }\end{array}$ & $\begin{array}{l}\text { Limites } \\
\text { Identité et capacité d'innovations } \\
\text { fortes }\end{array}$ & \\
\hline
\end{tabular}

\section{B. Propriétés d'un « système marginal »}

9 Trois caractéristiques majeures du système marginal sont successivement exposées : les contradictions entre le système et son environnement, l'invariance d'échelle et la 
complexité. Elles constituent les bases sur lesquelles toute recomposition doit être fondée.

\section{Les contradictions (Tableau 2)}

Que ce soient l'identité, l'organisation et l'intégration, les propriétés des systèmes territoriaux de marges présentent des contradictions si on les étudie du point de vue $d u$ système ou du point de vue de son environnement. Ainsi, si l'identité d'un système de marges est forte, proche du particularisme avec une qualité de vie préservée, l'insularité des mentalités et des habitudes de vie, cette identité n'en est pas moins fondée "sur le rejet » vis-à-vis de l'environnement. Le système bénéficie d'une certaine autonomie, mais il est aussi fortement dépendant des grands pôles économiques pourvoyeurs d'emplois et situés dans l'environnement. Enfin, si les limites du système sont précises, notamment du fait des contraintes physiques et du sentiment d'appartenance, elles ont évolué dans l'espace et dans le temps.

Tableau 2 : Le système marginal et son environnement

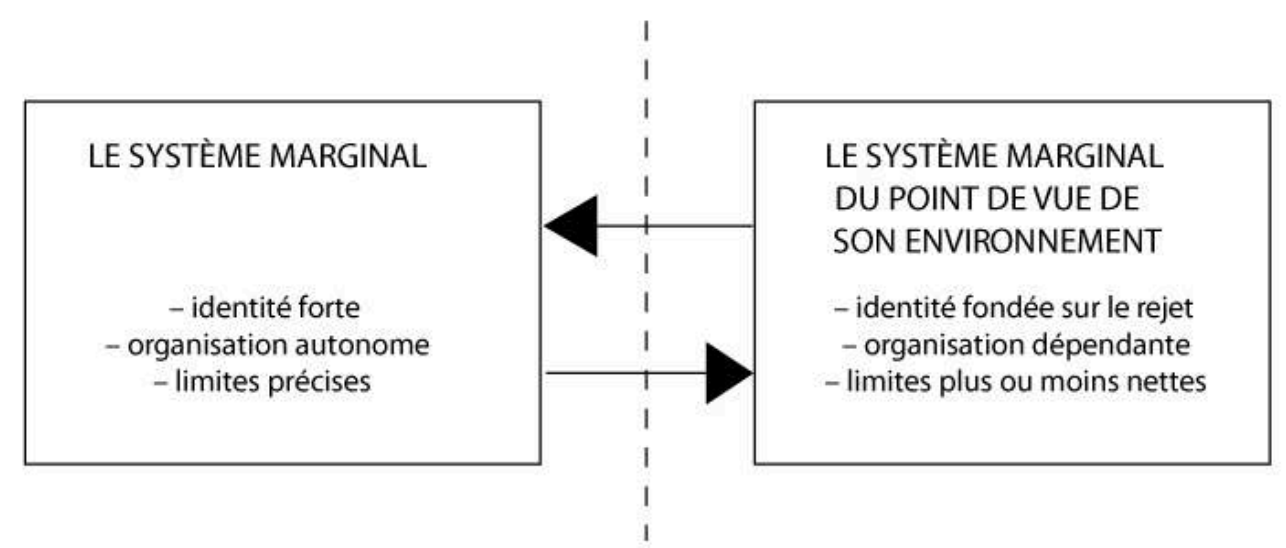

\section{L'invariance d'échelle (Tableau 3)}

11 Quelle que soit l'échelle territoriale, la marginalité génère approximativement la même configuration et la même géométrie. En effet, du local au régional, l'espace de marges apparaît avec une configuration particulière sous la forme d'une structure emboîtée qui conserve les mêmes propriétés à des échelles géographiques différentes, à la manière d'un système emboîté comme des "poupées russes", caractéristique d'une logique fractale. Le territoire Moselle-Alsace fournit une belle palette d'exemples de ce type où le point commun demeure celui de la marginalité. Ainsi, l'Est-Mosellan est un territoire de marges pour la Lorraine, le Pays de Bitche l'est aussi pour l'Est-Mosellan et enfin, le Pays du Verre et du Cristal constitue un territoire de marges pour le Pays de Bitche. 
Tableau 3 : L'invariance d'échelle

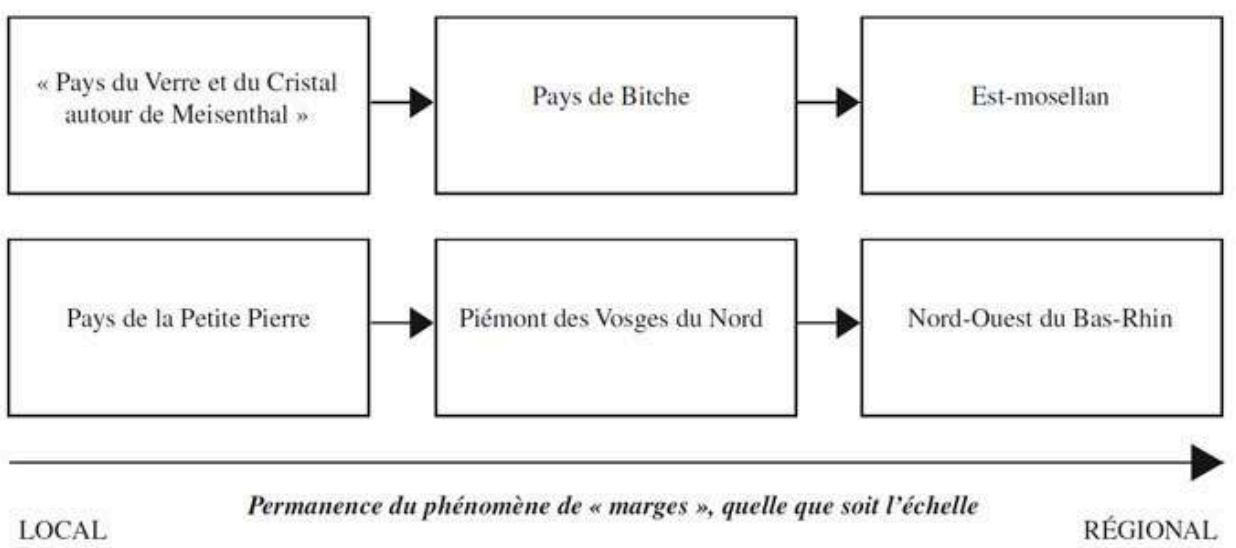

12 Ainsi, les résultats de travaux antérieurs (Ruffray S. de, 1999), montrent que l'espace de marges «Moselle-Est/Nord-Ouest du Bas-Rhin», observé à deux échelles géographiques différentes, apparaît toujours avec un cœeur, zone déprimée au centre et une bordure plus ouverte qui l'entoure. Son organisation dépend des pôles d'activités situés en périphérie ou à l'extérieur de l'espace.

\section{Les niveaux de complexité}

13 Sur le plan systémique, le système territorial de marges est un ensemble global dont les différents niveaux de complexité interne présentent des dysfonctionnements. L'exemple le plus significatif pour illustrer ce propos est celui de la hiérarchie urbaine dont nous soulignons ici trois caractéristiques majeures :

- l'absence de rayonnement significatif des villes moyennes;

- le « dopage » des petites villes, voire des centres locaux;

- le « nivellement » des centres (petites villes et centres locaux) qui prétendent tous à une position dominante sans en avoir les capacités réelles et entrent en compétition, voire en conflit insoluble, puisqu'aucun protagoniste n'a les forces suffisantes pour l'emporter.

Il en résulte un caractère fondamental majeur des systèmes territoriaux de marge, à savoir l'indépendance de la taille de la surface du territoire de cohérence (fondé à la fois sur l'identité, l'organisation et la capacité de projet) et du niveau de la hiérarchie urbaine auquel appartient son centre. Des travaux antérieurs (Rolland-May C., Ruffray S. de, 2001) sur l'espace de marges démontrent largement cette particularité. En effet, Saverne, Sarreguemines et Sarrebourg, qui représentent les niveaux de la hiérarchie les plus élevés de l'espace d'étude (ceux de ville-relais ou/et de ville moyenne), ont des territoires de cohérence particulièrement petits par leur étendue et réguliers par leur forme, et à peu près de la même taille que ceux de Sarralbe ou de Sarre-Union. Ainsi, l'espace d'étude apparaît sous la forme d'une mosaïque où tous les centres organisent le territoire de façon identique, nivelant toute forme de hiérarchie urbaine. 
Figure 1 : Une hiérarchie urbaine désarticulée

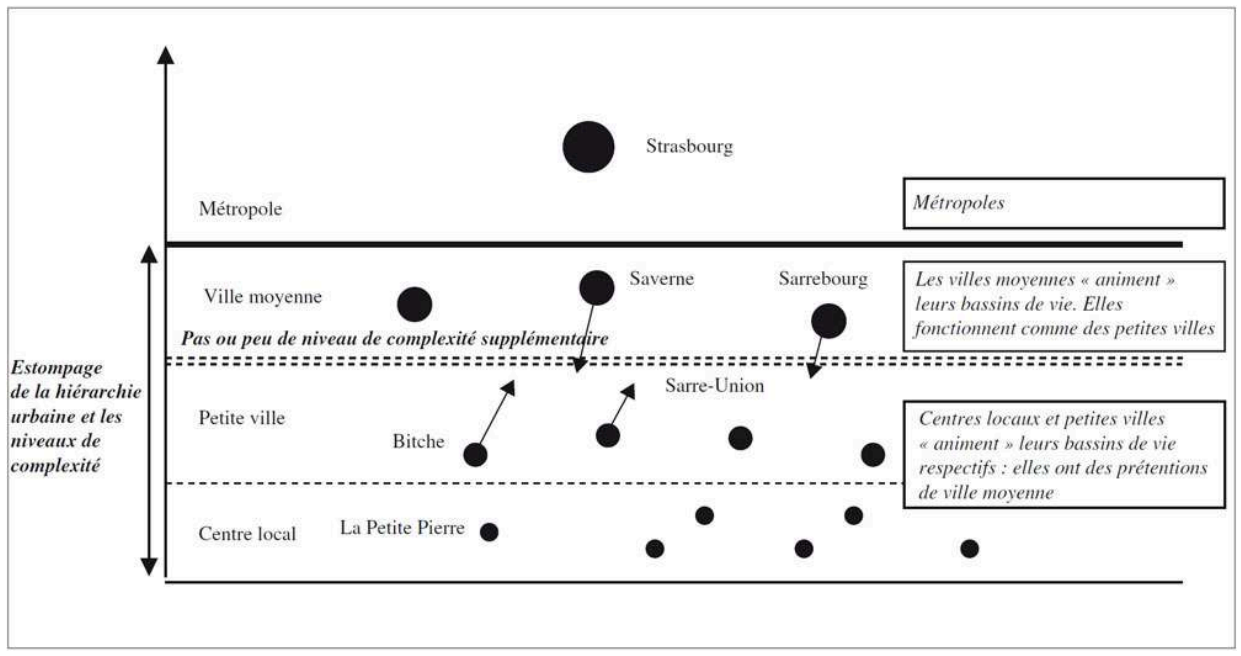

\section{Des caractéristiques du système marginal à la définition d'une recomposition marginale}

\section{De la recomposition des acteurs à la recomposition globale}

L'usage courant du terme de « recomposition des territoires » ne doit pas faire oublier que ce mot, dérivé du latin compositio, désigne l'action de former "un tout" en assemblant des éléments, et qui suppose implicitement un changement de qualité. Actuellement, la recomposition territoriale désigne souvent l'étude des structures intercommunales comme élément rassemblant plusieurs communes autour d'un projet de territoire qui tienne compte de l'évolution législative (mise en place des «pays » et des agglomérations, simplification des structures intercommunales).

Compte tenu de l'étymologie de ce terme de recomposition, l'usage actuel désigne une première étape nécessaire, mais non suffisante, qui concerne essentiellement l'échelle des acteurs. En effet, toute recomposition implique une vision plus large d'un point de vue spatial et une mise en convergence entre des aspects locaux et une vision globale. Cette mise en convergence repose essentiellement sur le fonctionnement des réseaux qu'ils soient économiques, sociaux ou plus globaux comme, par exemple, le réseau urbain.

Les deux visions ne sont pas contradictoires mais plutôt complémentaires, la recomposition à l'échelle des acteurs constituant la première étape indispensable, mais non suffisante pour fédérer les projets d'acteurs et pour permettre une action à une échelle spatiale plus large et plus complexe.

\section{Conséquences pour le territoire de marges}

Dans le cadre de ces définitions, la recomposition des territoires de marges doit permettre une meilleure intégration tout en respectant les propriétés intrinsèques de ces espaces au risque de fragiliser la pérennité du système. 
19 Ainsi, nous proposons l'idée qu'une recomposition territoriale marginale a pour caractéristique un modèle d'organisation de villes en réseau, ce dernier jouant l'interface entre les niveaux hiérarchiques urbains inférieurs et supérieurs.

Notre démarche est formalisée en deux étapes successives :

- la recomposition à l'échelle des acteurs, recomposition topologique qui concerne la dynamique locale, qui n'est pas spécifique aux espaces de marges ;

- une recomposition plus globale qui prenne en compte les caractéristiques majeures du système, à travers la présentation de modèles d'organisation marginale, fondés sur le rôle des réseaux de petites villes.

\section{La recomposition des territoires de marges, une dynamique double mais segmentée aux effets déterminants}

21 Si l'on considère une recomposition territoriale comme un regroupement «topologique » en structures intercommunales créées ou transformées, l'espace de marges présente une double dynamique spatiale. En effet, il existe une superposition de structures intercommunales, qui correspondent aux deux modèles de coopération intercommunale et qui agissent en interrelations, à deux échelles spatiales différentes. Le premier niveau de recomposition recouvre presque entièrement l'espace interrégional, qui se présente alors comme une mosaïque de "bassins de vie " centrés sur des petits centres ou des petites villes, où le facteur identitaire est important. Le deuxième niveau présente une structure intercommunale originale de coopération verticale, à travers le Parc Naturel Régional des Vosges du Nord, regroupant un certain nombre de partenaires institutionnalisés.

Cette double dynamique présente une vision segmentée aux effets déterminants pour l'ensemble de l'espace de marges.

\section{A. Une recomposition en mosaïque à l'échelle locale}

\section{Une mosaïque territoriale, où le facteur identitaire est prégnant}

Dans l'exemple étudié, à l'échelle locale, 86,7 \% des communes appartiennent à 19 structures intercommunales à fiscalité propre, comme le montrent la figure 2 et le tableau 4. Cette coopération horizontale est très ancienne puisque les structures sont presque toutes dites de "deuxième génération » c'est-à-dire qu'elles mènent des projets de développement dans le cadre de structures à fiscalité propre, résultats d'une évolution en fonction des opportunités ou des contraintes législatives, passant de syndicat en communauté de communes ou de syndicat en district. Sur la base de ce tableau, trois constats peuvent être énoncés :

- le faible nombre de communes et d'habitants regroupés par chacune des structures. En effet, chacune des structures regroupe en moyenne une quinzaine de communes, imposant au territoire une recomposition « de gestion » avec une économie d'échelle autour de petits projets d'acteurs locaux ; 
25 - l'absence de petite ville ou de bourg "porteur» pour certaines structures, comme la Communauté de communes du Pays du Verre et du Cristal ou celle de la vallée de la Bièvre ;

- un renforcement du dysfonctionnement de la hiérarchie urbaine. Les villes moyennes ne fédèrent pas plus les communes autour d'elles que les centres locaux. L'exemple de Sarrebourg est particulièrement démonstratif de ce constat puisque cette ville moyenne regroupe sept communes dans une communauté de communes nouvellement créée, loin de fédérer son arrière-pays où d'autres communautés de communes ont vu le jour, comme celle du Pays de Fénétrange.

Tableau 4 : Les structures intercommunales dans l'espace de marges

\begin{tabular}{|c|c|c|c|c|}
\hline $\begin{array}{c}\text { Structure } \\
\text { intercommunale }\end{array}$ & $\begin{array}{l}\text { Date de } \\
\text { création }\end{array}$ & $\begin{array}{c}\text { Nombre } \\
\text { de communes } \\
\text { et thombre } \\
\text { d'habitants }\end{array}$ & $\begin{array}{c}\text { Commune } \\
\text { principale } \\
\text { «portant " la } \\
\text { structure }\end{array}$ & $\begin{array}{l}\text { Remarques concernant } \\
\text { l'évolution }\end{array}$ \\
\hline $\begin{array}{l}\text { C. de C de Bitche } \\
\text { et environs }\end{array}$ & 1994 & $\begin{array}{c}14 \\
10889 \text { hab }\end{array}$ & Bitche & Ancien SIVOM de Bitche avec départ des communes \\
\hline $\begin{array}{l}\mathrm{C} \text { de } \mathrm{C} \text { du Pays du Verre } \\
\text { et du Cristal }\end{array}$ & 1996 & $\begin{array}{l}7 \\
8663 \text { hab }\end{array}$ & Meisenthal & Ancien sivom de Lemberg \\
\hline $\begin{array}{l}\text { C. de C du Pays } \\
\text { de Fenetrange }\end{array}$ & 1997 & $\begin{array}{l}20 \\
6498 \text { hab }\end{array}$ & Fenetrange & \\
\hline $\begin{array}{l}\text { C. de C du Pays } \\
\text { de Phalsbourg }\end{array}$ & 1994 & $\begin{array}{l}26 \\
16709 \text { hab }\end{array}$ & Phalsbourg & Limites du canton de Phalsbourg \\
\hline C. de $C$ des deux Sarres & 1998 & $\begin{array}{c}18 \\
6670 \text { hab }\end{array}$ & Lorquin & Limites du canton de Lorquin \\
\hline $\begin{array}{l}\text { C. de } \mathrm{C} \text { de Rohrbach } \\
\text { les Bitche }\end{array}$ & 1993 & $\stackrel{9}{9512 \text { hab }}$ & Rohrbach les Bitche & \\
\hline $\begin{array}{l}\text { C. de C de l'agglomération } \\
\text { de Sarrebourg }\end{array}$ & 1996 & $\begin{array}{l}7 \\
19159 \text { hab }\end{array}$ & Sarrebourg & \\
\hline District de Sarreguemines & 1991 & $\begin{array}{c}21 \\
45066 \text { hab }\end{array}$ & Sarreguemines & \\
\hline $\begin{array}{l}\text { C. de } C \text { de la Vallée } \\
\text { de la Biêre }\end{array}$ & 1997 & $\begin{array}{c}11 \\
8050 \text { hab }\end{array}$ & Troisfontaines & \\
\hline District de Volmunster & 1991 & $\begin{array}{c}16 \\
6030 \text { hab }\end{array}$ & Volmunster & Limites du canton de Volmunster \\
\hline $\begin{array}{l}C \mathrm{Cde} C \text {. du Pays de } \\
\text { Sarre-Union }\end{array}$ & 1998 & $\begin{array}{c}13 \\
11405 \text { hab }\end{array}$ & Sarre-Union & Ancienne $C$ de C de l'alsace Bossue cráée en 1995 \\
\hline C. de C de IAlsace Bossue & 1998 & $\begin{array}{c}32 \\
13313 \text { hab }\end{array}$ & Drulingen & Ancienne $C$ de $C$ de l'Alsace Bossue créée en 1995 \\
\hline $\begin{array}{l}\text { C. de C de la vallée } \\
\text { de la Saver }\end{array}$ & 1993 & $\begin{array}{c}17 \\
9813 \text { hab }\end{array}$ & Woerth & \\
\hline $\begin{array}{l}\text { C. de C du Pays } \\
\text { de la Petite Pierre }\end{array}$ & 1992 & $\begin{array}{c}19 \\
9445 \text { hab }\end{array}$ & La Petite Pierre & \\
\hline $\begin{array}{l}\text { C. de C du Pays } \\
\text { de Wissembourg }\end{array}$ & 1994 & $\begin{array}{c}8 \\
12290 \text { hab }\end{array}$ & Wissembourg & \\
\hline District du Pays de Hanau & 1992 & $\begin{array}{c}18 \\
15946 \text { hab }\end{array}$ & Bouxwiller et Ingwiller & \\
\hline C. de C de Pechelbronn & 1992 & $\begin{array}{c}5 \\
3699 \text { hab }\end{array}$ & Pechelbronn & Ex-SIVOM de Pechelbronn \\
\hline C. de C.du Pays de Saverne & 1997 & $\begin{array}{c}18 \\
24971 \text { hab }\end{array}$ & Saveme & Ex-district de Saverne \\
\hline $\begin{array}{l}\text { Syndicat de dévt du canton } \\
\text { de Niederbronn les Bains }\end{array}$ & 1986 & $\begin{array}{l}20 \\
24479 \text { hab }\end{array}$ & Niederbronn & \\
\hline
\end{tabular}


Figure 2 : Les structures intercommunales dans l'espace de marges Moselle Est / Nord-Ouest du Bas-Rhin

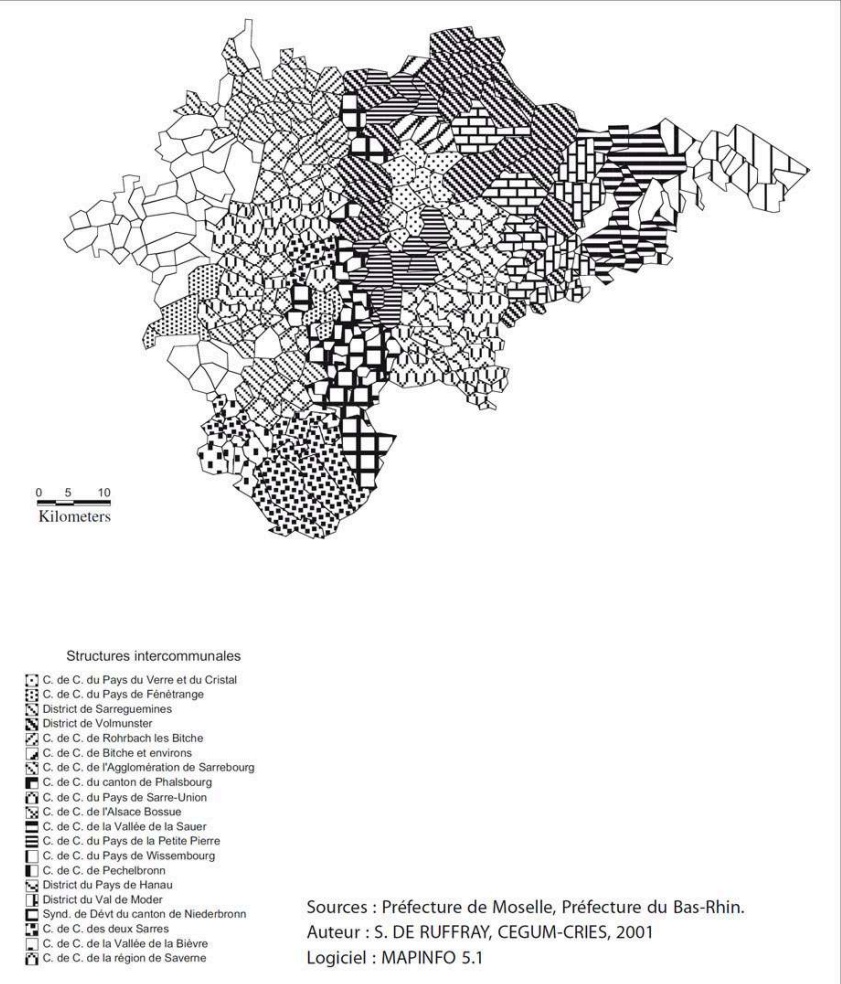

\section{Une multitude de projets d'acteurs}

Suivant les propositions de C. Rolland-May (2000), le territoire de marges est caractéristique d'un niveau stratégique de base dynamique, constitué par les acteurs et les réseaux d'acteurs, indispensable pour une dynamique territoriale locale, mais qui entraîne le renforcement de la mosaïque territoriale.

Les acteurs, que ce soient des individus, des élus, des associations ou des acteurs économiques, sont ancrés dans le territoire, marqués par une appartenance forte et détiennent de l'information, même sectorielle. L'ensemble de ces acteurs locaux, qui forment le réseau d'acteurs, constitue l'assise indispensable des dynamiques territoriales et des formes élémentaires d'organisation du territoire. Grâce à cette énergie élémentaire, les réseaux d'acteurs peuvent initier des projets de développement local et concevoir à leur échelle des réalisations conformes à leur souhait. Ainsi, chacune des structures intercommunales précédemment décrites possède un projet de développement centré sur un ou plusieurs secteurs concernés : le développement économique (création d'une ZAC...), l'habitat (réalisation d'une $\mathrm{OPAH}$, développement de logements locatifs...), l'agriculture et l'artisanat local (création d'un marché des produits du terroir, réalisation d'une ORAC...), le social (création d'une maison pour la petite enfance...), le tourisme (Maison de l'eau et de la rivière, Centre international de l'art verrier...).

Le territoire de marges est ainsi concerné par une multitude de projets, portés par un réseau spécifique d'acteurs, à travers chaque structure intercommunale... Au niveau global, le territoire apparaît comme une mosaïque de "micro-projets», parfois 
redondants comme dans le cas des zones d'activités, mais signes d'une dynamique locale forte, articulée autour des bassins de vie.

\section{Conséquences}

30 Ainsi, la multitude des structures intercommunales au sein de l'espace de marges étudié montre que le facteur identitaire demeure prégnant autour des projets d'acteurs locaux. Sur l'ensemble de cet espace, la recomposition demeure une mosaïque à l'échelle locale, sans organisation et sans logique globale.

\section{B. Une recomposition inachevée à l'échelle interrégionale}

Outre la recomposition en structures intercommunales, le Parc Naturel Régional des Vosges du Nord constitue une forme de recomposition à l'échelle interrégionale. Créé pour préserver les richesses naturelles de ce territoire, le SYCOPARC constitue une structure fédératrice des dynamismes locaux, en stimulant et orientant les projets liés à l'environnement et au développement du territoire. Compte tenu de son fonctionnement et de ses compétences, le Parc est un lieu de regroupement de projets et d'acteurs locaux plus qu'un lieu de mise en cohérence ou éventuellement d'émergence de projets fédérateurs, véritables vecteurs d'une recomposition territoriale plus globale.

\section{Une structure fédératrice}

A l'échelle interrégionale, l'espace est classé « Réserve de Biosphère " par l'Unesco avec la présence du Parc Naturel Régional des Vosges du Nord. Animé par le SYCOPARC, Syndicat de Coopération du Parc, la Charte constitue un projet global d'aménagement, de protection et de développement des territoires dans le respect des patrimoines naturels et culturels locaux. Cette coopération est verticale puisqu'elle regroupe des partenaires institutionnels comme l'Etat, les Régions Lorraine et Alsace, les Départements de la Moselle et du Bas-Rhin, les Etablissements Publics de Coopération Intercommunale et les communes.

D'un point de vue spatial, le Parc s'étend dans la partie nord du massif vosgien, jusqu'aux villes moyennes de Sarreguemines, Sarrebourg, Saverne et Haguenau, qui constituent des « villes-portes ».

Le projet regroupe quatre axes différents avec la préservation de l'espace et de l'environnement, le développement économique et social, la protection et la mise en valeur du patrimoine bâti et l'animation. A l'origine plus centré sur la préservation des espaces naturels et de la biosphère, il se préoccupe de plus en plus du développement des territoires, ce qui pose le problème de l'articulation de ses actions avec les structures intercommunales existantes.

\section{Une cohérence d'échelle et d'action difficile à mettre en œuvre}

Deux exemples peuvent illustrer la difficile mise en œuvre de la cohérence entre l'action menée et l'échelle spatiale de pertinence, entre l'échelle de proximité (les structures intercommunales) et l'échelle globale (le Parc Naturel Régional des Vosges du Nord). Le SYCOPARC, malgré son rôle fédérateur, n'a pas pu lutter contre la mise en place des nombreuses zones d'activités par les structures intercommunales de cet espace 
interrégional. En concurrence les unes avec les autres, ces ZAC illustrent l'absence de mise en cohérence de projets économiques locaux.

Pour lutter contre la fermeture des paysages et le problème posé par l'absence de l'agriculture sur des secteurs difficiles à exploiter comme les milieux « en friches » des fonds de vallées, les collectivités locales ont mis en place une solution écologique. Pour cela, des «Highland Cattle», race écossaise de bovins, capables de subsister et de se nourrir de végétaux propres aux milieux humides « en friches ", sont installés dans les parcelles dont ils broutent l'herbe. Cette action, à l'instigation du Parc, a des impacts positifs sur l'ouverture des paysages mais entraîne des coûts supplémentaires pour les structures intercommunales. En effet, le Parc apporte son soutien technique et scientifique (mise à disposition des animaux, suivi sanitaire et technique du programme et des conséquences écologiques du pâturage), mais la gestion concrète, tout comme le devenir des animaux, demeurent à la charge des communes ou des structures intercommunales (mise en place des clôtures, surveillance et nourriture des animaux,...)

\section{Une recomposition spatiale limitée}

Malgré la présence du Parc Naturel Régional des Vosges du Nord qui fédère les dynamiques locales, la recomposition des territoires à l'échelle interrégionale est limitée sur le plan thématique et sur le plan des acteurs. Ainsi, il constitue un lieu de rencontres entre les élus ou les acteurs des structures intercommunales, un regroupement de projets locaux plus qu'un lieu de mise en cohérence ou éventuellement d'émergence de projets fédérateurs. Citons l'exemple de la thématique touristique pour illustrer ce propos. Le projet du Parc a été de créer un réseau d'offices du tourisme regroupant les offices des structures intercommunales sans initier un projet touristique global.

\section{Des effets induits déterminants}

39 La recomposition en mosaïque à l'échelle locale et la recomposition inachevée à l'échelle interrégionale présentent trois effets majeurs déterminants. Ils concernent à la fois l'écrasement de la hiérarchie urbaine, les logiques de proximité et les logiques de relations avec les villes.

Le premier effet d'une recomposition territoriale unique, à l'échelle des acteurs, est de négliger le rôle fondamental de la hiérarchie urbaine dans l'organisation globale de l'espace. En effet, chaque centre, quelle que soit sa taille et son importance dans la hiérarchie urbaine, joue un rôle fédérateur équivalent pour un ensemble plus ou moins vaste de communes environnantes. Chacune de ces structures intercommunales joue le même rôle, regroupant les mêmes compétences, conformément aux dispositions législatives. La recomposition entraîne de ce fait un écrasement de la hiérarchie urbaine.

41 Des travaux antérieurs ont mis en évidence des territoires de cohérence pour 16 centres de ce territoire d'étude (Rolland-May C., Ruffray S. de, 2001). Comprise entre 0 et 100 , chaque valeur représente l'appartenance d'une commune à un centre donné en combinant huit indicateurs, fondés sur les notions d'identité, d'organisation et de capacité à porter un projet. Chaque commune de la zone d'étude a une valeur 
d'appartenance pour chacun des 16 centres, qu'il est possible de comparer, notamment pour étudier les logiques de proximité.

Dans un espace de marges, les logiques spatiales d'attractivité par rapport à un centre situé à proximité ne jouent plus, mis à part pour la valeur d'appartenance maximum à un centre. Cela confirme combien la recomposition à l'échelle des acteurs sur un territoire précis et délimité marque les logiques territoriales et les dysfonctionnements de la hiérarchie urbaine. Deux exemples peuvent montrer combien les logiques de proximité ne sont pas respectées dans un espace de marges (Fig. 3). Ainsi, la commune de Rahling est rattachée au territoire de cohérence de Bitche avec une valeur d'appartenance de 26, 64 (cette valeur même faible correspond à la valeur d'appartenance maximum). Par ordre décroissant, les logiques de proximité ne jouent plus puisque les valeurs d'Ingwiller $(20,75)$, de la Petite Pierre $(20,54)$ et de Wissembourg $(17,74)$ sont supérieures aux valeurs d'appartenance de Drulingen $(15,67)$, Sarralbe $(14,38)$ et Sarre-Union $(12,75)$, pourtant plus proches de Rahling. Le deuxième exemple significatif, est celui de la commune de Vahl les Benestroff, à l'Ouest d'Albestroff. En effet, cette commune a une valeur d'appartenance maximum à Albestroff, avec 15,18; puis respectivement à Dabo et Drulingen (avec 12,36 et 12,16) alors que les valeurs pour Fenetrange et Sarralbe, plus proches, sont de 4,08 et 9,68 .

Figure 3 : Les dysfonctionnements des logiques des proximité

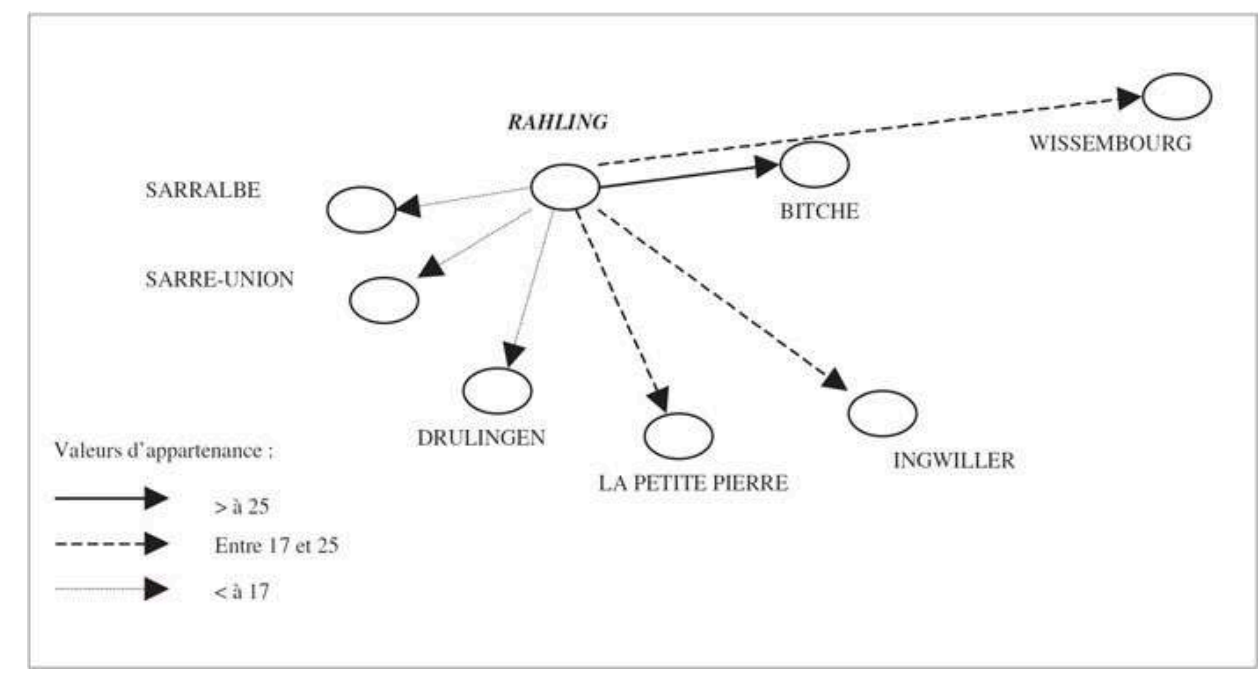

\section{(1)}

habitants avec lesquelles elle avait des relations et pourquoi. Neuf raisons majeures sont proposées en guise de réponse : « des entreprises de notre commune travaillent ou sont en relation avec des entreprises de la ville", "la ville offre des commerces dont notre commune ne dispose pas", «la ville offre des services privés non commerciaux dont la commune ne dispose pas", "la ville offre des emplois", "la ville offre des possibilités de loisirs et de culture dont notre commune ne dispose pas ", " la ville offre des possibilités de formation", "nous nous sentons appartenir à une même communauté sans qu'il y ait forcément de raison objective », « autres raisons »... 
45 Ainsi, sur 250 réponses, Strasbourg est citée 187 fois comme étant une des villes avec laquelle la commune a des relations, loin devant les villes moyennes de Saverne, Sarrebourg et Sarreguemines. Ce court-circuit vers les métropoles régionales (Metz et Strasbourg) est beaucoup plus marqué pour Strasbourg, du fait de la proximité de la capitale alsacienne avec l'espace d'étude.

46 A l'échelle des acteurs, la recomposition du territoire se présente sous la forme d'une mosaïque de "bassins de vie", de taille identique, quel que soit le centre local, porteur de projets locaux. Conformément à notre préoccupation initiale, cette recomposition spatiale topologique est insuffisante et nous proposons des modèles de recomposition marginale, qui concernent à la fois les logiques territoriales et résiliaires.

\section{Approche de modèles de recomposition « marginale »}

\section{A. Présentation générale}

Compte tenu des objectifs, la recomposition territoriale des territoires de marges doit permettre une meilleure intégration de ces espaces dans leur environnement, en ayant une logique d'organisation globale et en respectant les propriétés intrinsèques de ces espaces.

48 Nous proposons la définition d'une recomposition territoriale marginale fondée sur le fonctionnement particulier du réseau urbain comme interface entre la logique territoriale et la logique résiliaire.

49 Nous proposons ici deux scenarii de recomposition territoriale marginale, sous la forme de modèles d'organisation de villes en réseau :

50 - le premier, plus utopique, propose un «surclassement » du réseau des petites villes en une ville moyenne ;

51 - le second, plus pragmatique, comporte deux volets : d'une part, un réseau de petites villes joue le rôle d'interface entre le territoire et les villes moyennes environnantes; d'autre part et plus généralement, il propose une hiérarchie urbaine, fortement connexe.

\section{B. Le modèle de "surclassement » : le rêve du réseau de petites villes (Fig. 4)}

52 Compte tenu des dysfontionnements de la hiérarchie urbaine et du dynamisme des acteurs locaux, nous proposons un premier modèle de recomposition, fondé sur le « surclassement » : un réseau de petites villes fonctionne en se partageant les fonctions d'une ville moyenne. Les contraintes sont multiples et concernent la gestion, difficile à mener entre les petites villes toutes éloignées géographiquement et la répartition des fonctions de la ville moyenne entre les petites villes, fonctions qui doivent elles-mêmes être bien identifiées. 
Figure 4 : Le modèle d'organisation pour une recomposition marginale : le réseau de petites villes joue le rôle d'une ville moyenne

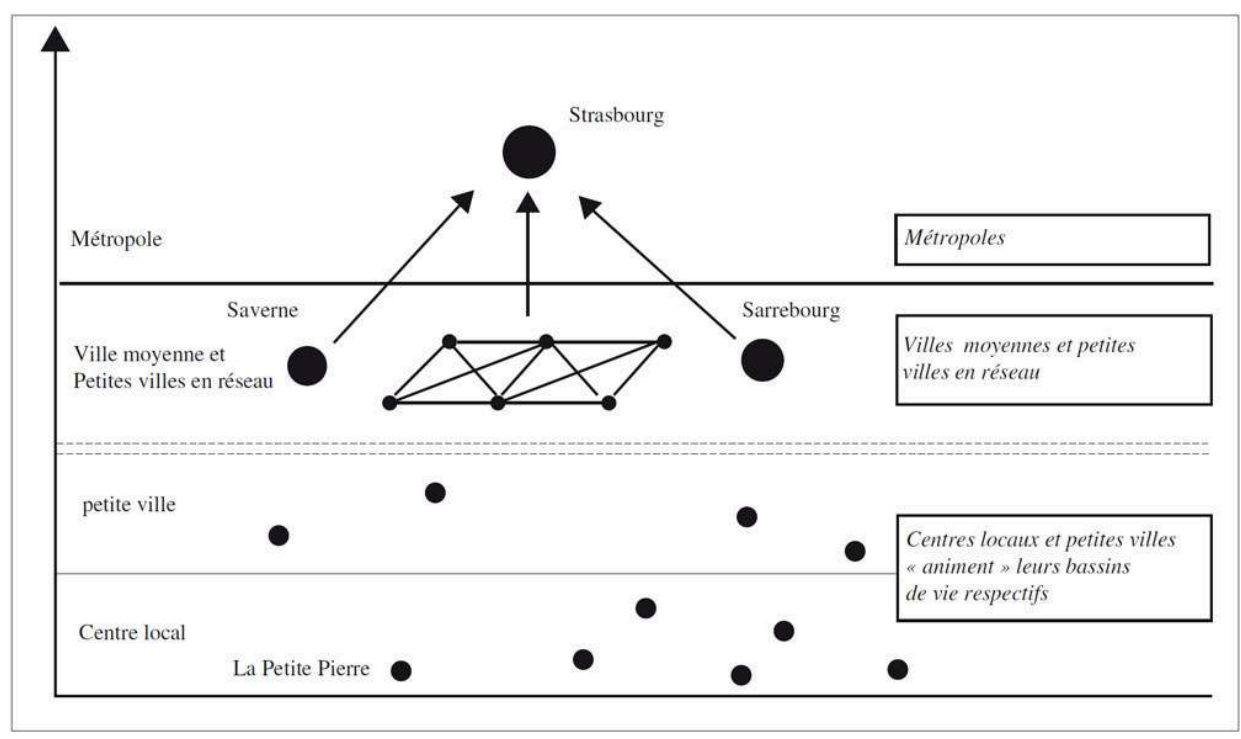

Des aspects positifs et négatifs de ce scénario peuvent être dégagés. Le réseau de villes, comme association de petites villes, est presque toujours réalisable, du moins en théorie, puisque ce maillon de la hiérarchie urbaine irrigue l'ensemble des territoires. Il suppose que l'association de petites villes peut faire émerger les qualités intrinsèques d'une ville moyenne en jouant le rôle de fédérateur des petits projets locaux, le relais vis-à-vis de la métropole, la mise en cohérence et l'émergence des projets globaux; ce qui est à l'opposé de toute vision systémique, toujours soucieuse de montrer qu'un ensemble n'est pas qu'une somme d'éléments.

En ce qui nous concerne, le réseau de petites villes peut regrouper les villes de Bitche, Bouxwiller, Sarre-Union et Sarralbe et jouer le rôle d'une ville moyenne au cœur du massif vosgien.

Si la compétition entre ces petites villes risque d'être atténuée par ce scénario, les effets prévisibles sont nuancés. En effet, les dysfonctionnements de la hiérarchie urbaine ne sont pas pris en compte, notamment celui des villes moyennes, qui, malgré leur taille, organisent le territoire de la même façon que les petites villes.

\section{Modèle de recomposition territoriale : le « réseau de petites villes » devient interface vis-à-vis des villes moyennes}

Premier volet du second scénario de recomposition territoriale, ce modèle plus pragmatique propose un réseau de petites villes comme interface entre le territoire et les villes moyennes environnantes. Ainsi, cinq niveaux urbains apparaissent sur la figure 5 avec un niveau intermédiaire, pivot de la recomposition marginale : un réseau de petites villes. L'association de ces petites villes a essentiellement pour rôle de jouer l'interface entre les centres locaux d'une part et les villes moyennes d'autre part. Il permet de répondre aux dysfonctionnements de la hiérarchie urbaine et à la configuration particulière d'un territoire de marges. Contrairement au modèle précédent, les petites villes en réseau ne se partagent pas les fonctions d'une ville 
moyenne, mais elles ont un rôle particulier d'interface pour fédérer le monde rural qui les entoure et les villes moyennes environnantes.

Figure 5 : Le modèle d'organisation pour une recomposition marginale : le réseau de villes interface avec les villes moyennes

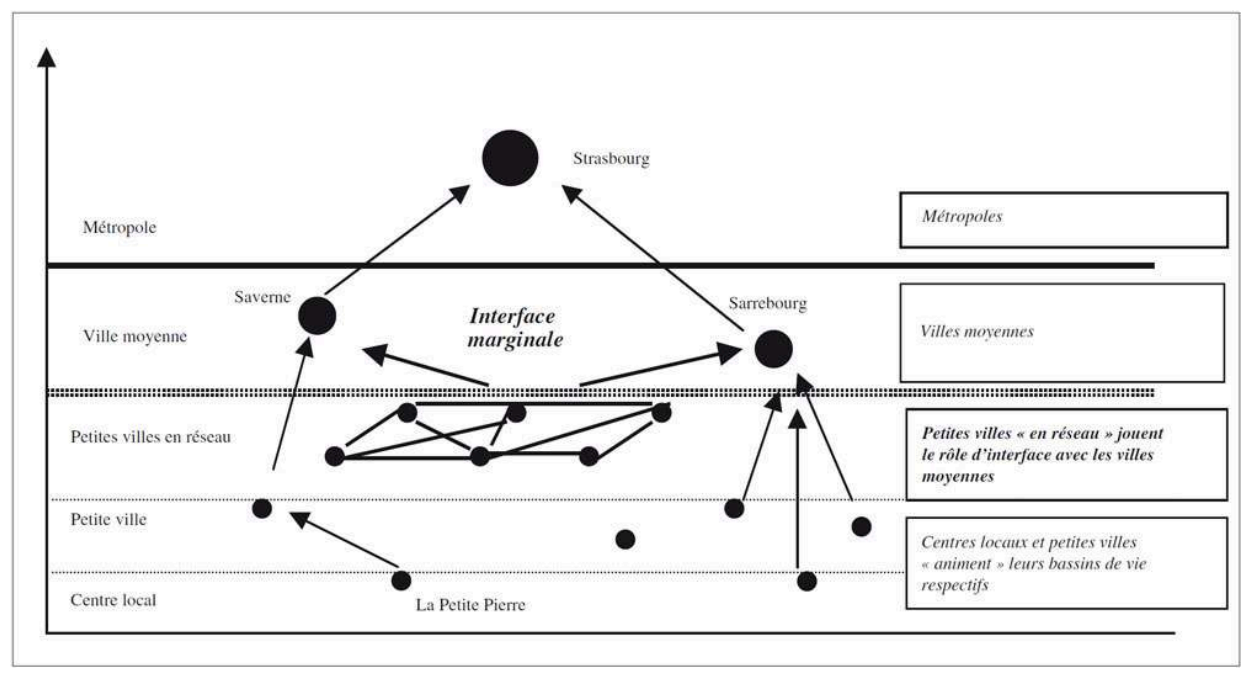

Ce scénario présente un certain nombre d'avantages, dont celui de renforcer les niveaux urbains dans leurs rôles respectifs. Ainsi, le réseau de petites villes, sans prétendre remplacer une ville moyenne, permet à cette dernière de jouer son rôle visà-vis des territoires environnants. A l'inverse, ce regroupement de petites villes leur permet aussi de "mieux négocier » vis-à-vis de la ville moyenne, plus à l'écoute d'un réseau fédéré de petites villes que d'un centre local isolé. Ainsi, chacun des niveaux de la hiérarchie urbaine est renforcé.

\section{Modèle de recomposition territoriale : une hiérarchie urbaine de forte connexité}

Deuxième volet du scénario de recomposition territoriale, ce modèle généralise le réseau de petites villes comme interface entre le territoire et les villes moyennes environnantes, en proposant une hiérarchie urbaine de forte connexité. Ainsi, un niveau intermédiaire « interface en réseau » entre les niveaux inférieurs et supérieurs devient le pivot de toute forme de recomposition marginale.

Sur la figure 6, deux interfaces marginales ont été représentées :

- l'une concerne un réseau de petites villes, interface entre les centres locaux d'une part et les villes moyennes d'autre part ;

- l'autre concerne un réseau de centres locaux, interface entre le territoire et les petites villes. 
Figure 6 : Le modèle d'organisation pour une recomposition marginale : une hiérarchie urbaine de forte connexité

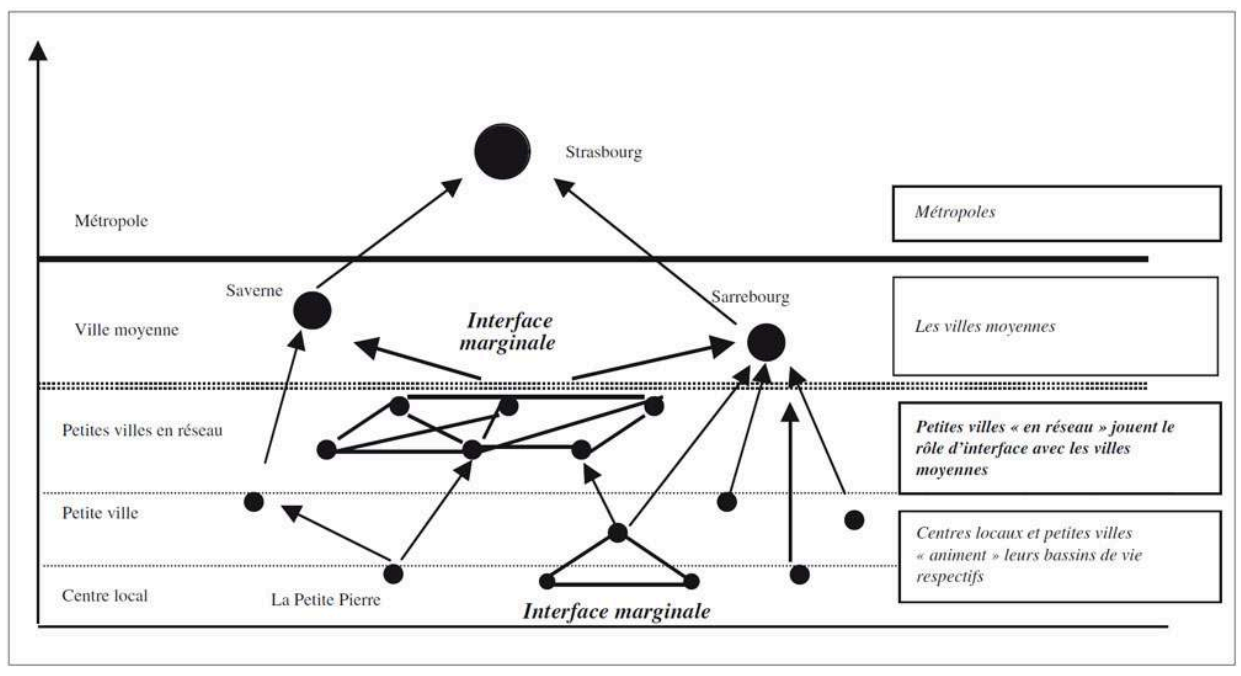

L'application de ce modèle de hiérarchie urbaine de forte connexité à notre espace de marges présente plusieurs niveaux urbains :

- Sarrebourg et Saverne représentent les deux villes moyennes ;

- un réseau de petites villes, première interface marginale, est formée de la réunion des petites villes comme Bitche, Sarre-Union, Sarralbe, Drulingen, Bouxwiller et Ingwiller ;

- un réseau de centres locaux, deuxième interface marginale, est constitué par l'association de Diemeringen et Wingen-sur-Moder, à proximité de Drulingen.

\section{Conclusion et perspectives}

61 Dans le cadre de l'espace d'étude, le modèle de recomposition marginale permet de résoudre les dysfonctionnements de la hiérarchie urbaine et l'invariance de la marginalité, quelle que soit l'échelle spatiale d'étude. Le deuxième modèle, qui repose sur un réseau de villes jouant l'interface, est celui qui présente le moins de contraintes sur le plan conceptuel et sur le plan pratique.

A l'échelle interrégionale, une entité originale apparaît formée de l'union de trois entités (Fig. 7) : une première autour de la ville moyenne de Sarrebourg et de son arrière-pays, une deuxième autour de la ville moyenne de Saverne et enfin, une troisième entité "marginale " composée d'un réseau de petites villes comme Bitche, Sarre-Union, Sarralbe, Bouxwiller et Ingwiller, jouant le rôle d'interface.

Ces trois entités peuvent jouer un rôle dans la constitution des "pays », prévus dans le cadre de la Loi d'orientation pour l'aménagement et le développement durable du territoire. Plus grands que les structures intercommunales actuelles, les pays se constitueront autour des villes moyennes qui devront s'appuyer sur les petites villes en réseau pour jouer leur rôle vis-à-vis du monde rural environnant. 
Figure 7 : L'espace de marges : une entité marginale recomposée

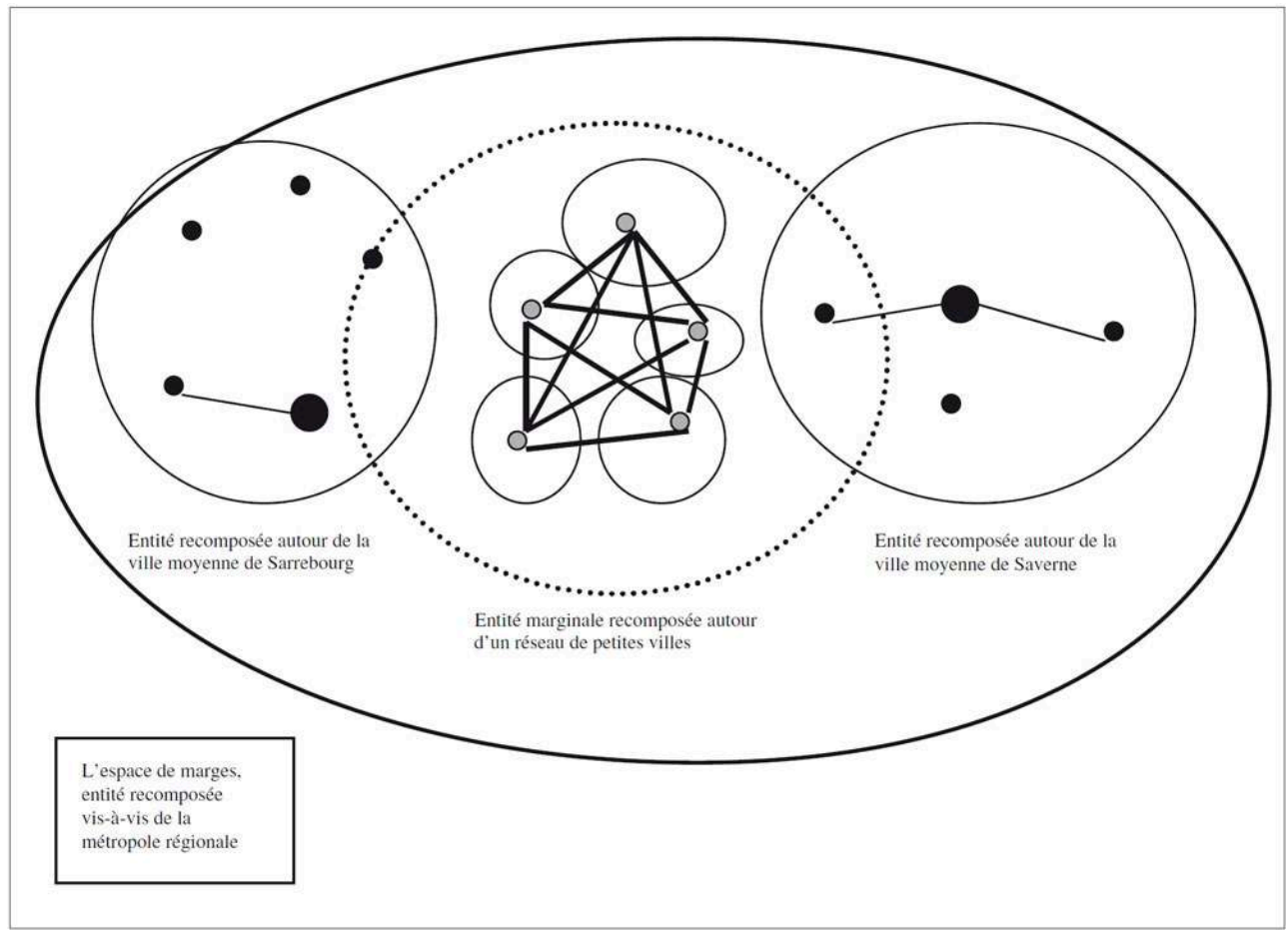

\section{BIBLIOGRAPHIE}

Brunet R., Ferras R., Thery H. - Les mots de la géographie, Dictionnaire critique, Paris, Collection dynamique des territoires, GIP Reclus, La documentation française, 1992, $470 \mathrm{p}$.

DATAR, « Territoires 2020 ». - Revue d'études et de prospective, $\mathrm{n}^{\circ}$ 1, La Documentation française, Paris, 2000, $101 \mathrm{p}$.

INSEE, Revue Economie Lorraine, nº 128 et n 151, février 1994 et mars 1996

Parc Naturel Regional Des Vosges Du Nord. - «Charte », document dactylographié, 1993, 101 p.

Préfecture De La Région Alsace. - «Les forces et les fragilités socio-économiques du territoire alsacien entre 1990 et 1996 », 1998, 8 p. et cartes en annexes.

Reynaud A. - «Centre et périphéries », p. 583-599, Encyclopédie de la géographie, sous la direction d'A. Bailly, R. Ferras, D. Pumain, Paris, Economica, 1992, 1132 p.

Rolland-May C. - «L'évaluation des territoires : concepts, modèle et méthodes », Hermès, Paris, 2000, $381 \mathrm{p}$.

Rolland-May C. - «Périphéries, bordures, marges territoriales : sous les mots, les concepts », Collection RITMA, Regards croisés sur les territoires de marge(s), à paraître en 2001, Presses Universitaires de Strasbourg, 16 p. 
Rolland-May C., Ruffray S. de. - « Vers une évaluation globale des espaces de marges par la mise en convergence des territoires et des réseaux. L'exemple de la Moselle-Est et du Nord-Ouest du Bas-Rhin ", Collection RITMA, Regards croisés sur les territoires de marge(s), à paraître en 2001, Presses Universitaires de Strasbourg, $23 \mathrm{p}$.

Rosnay J. de. - « Le macroscope, vers une vision globale », Points Essais, Ed. Le Seuil, Paris, 1975, 346

p.

Ruffray S. de. - «Mise en évidence de l'organisation spatiale d'un espace de marges. L'exemple de l'interface Moselle Est - Alsace du Nord-Ouest », Revue Mosella, Tome XXIV, No 3-4,1999, pp.

41-64.

Ruffray S. de. - «mise en évidence de l'organisation spatiale des deux territoires d'étude ", Collection RITMA, Regards croisés sur les territoires de marge(s), à paraitre en 2001, Presses Universitaires de Strasbourg, 18 p.

\section{RÉSUMÉS}

Dans le contexte des dynamiques spatiales actuelles, cet article propose une réflexion sur la notion de recomposition territoriale dans les espaces de marges. Il a pour objectif de présenter des modèles de recomposition "marginale", fondés sur des interfaces particulières, sous la forme de réseaux de villes pour répondre aux propriétés particulières des territoires de marges. L'application concerne l'espace interrégional, constituant l'interface entre les confins orientaux de la Moselle et nord-occidentaux du Bas-Rhin.

This paper examines the principle of territorial recomposition in peripheral locations, in the context of spatial dynamics. Its objective is to present various models of marginal recomposition based on particular situations in which networks of cities respond to the particular properties of marginal territories. A case study of an interregional area lying between the eastern borders of the Moselle and northwestern Bas-Rhine is presented.

Im Zusammenhang mit den aktuellen Raumdynamismen bietet dieser Artikel eine Überlegung über den Begriff der territorialen Neuordnung in marginalen Bereichen. Er hat das Ziel, Modelle einer « marginalen » Neuordnung vorzustellen, basierend auf speziellen « Schnittstellen » in der Form von Städtenetzen, um den besonderen Eigenheiten randlicher Territorien gerecht zu werden. Die Anwendung betrifft den interregionalen Raum, Schnittstellen zwischen dem östlichen Grenzbereich des Departements Moselle und dem nordwestlichen des Departements Bas-Rhin.

\section{INDEX}

Schlüsselwörter : Modell, Randbereiche, Städtenetz, territoriale Neuordnung

Mots-clés : marges, modèle, recomposition marginale, villes en réseau

Keywords : marginal recomposition, marginal territories, model, network of cities

\section{AUTEUR}

SOPHIE DE RUFFRAY

CEGUM-CRIES - Université de Metz - Ile du Saulcy 57045 Metz Cedex 1 